CERN-TH.6803/93

SLAC-PUB-6054

UCLA/93/TEP/4

February, 1993

\title{
One-Loop Corrections to Five-Gluon Amplitudes
}

\author{
Zvi Bern \\ Department of Physics \\ University of California, Los Angeles \\ Los Angeles, CA 90024 \\ bern@physics.ucla.edu \\ Lance Dixon* \\ Stanford Linear Accelerator Center \\ Stanford, CA 94309 \\ lance@slacvm.slac.stanford.edu \\ and \\ David A. Kosower ${ }^{\dagger}$ \\ Theory Division \\ CERN \\ CH-1211 Geneva 23 \\ Switzerland \\ kosower@nxth02.cern.ch
}

\begin{abstract}
We present the one-loop helicity amplitudes with five external gluons. The computation employs string-based methods, new techniques for performing tensor integrals, and improvements in the spinor helicity method.
\end{abstract}

Submitted to Physical Review Letters

${ }^{\ddagger}$ Research supported by the Texas National Research Laboratory Commission grant FCFY9202.

${ }^{\star}$ Research supported by the Department of Energy under grant DE-AC03-76SF00515.

${ }^{\dagger}$ On leave from the Centre d'Etudes of Saclay, F-91191 Gif-sur-Yvette cedex, France. 
Calculations beyond the leading order in quantum chromodynamics are important to refining our understanding of known physics in present-day and future collider experiments, such as the Tevatron or the SSC and LHC. In jet physics, next-to-leading order calculations are important to curing several deficiencies of their leading-order counterparts: the strong spurious dependence on the renormalization scale; the lack of sensitivity to the jet resolution parameters, namely the minimum transverse energy and the jet cone size; and the absence of a warning about dangerous infrared logarithms. The one-loop corrections to matrix elements for $2 \rightarrow 2$ processes in QCD, a key ingredient of the next-to-leading order calculations of inclusive-jet and two-jet cross-sections and distributions, were computed by Ellis and Sexton [1]. To go beyond these cross-sections, whether to higher orders for two-jet cross-sections, or to next-to-leading order for three-jet cross-sections and distributions, requires the computation of the one-loop corrections to the $2 \rightarrow 3$ matrix elements. At hadron colliders, the QCD coupling $\alpha_{s}$, and the manner of its running, can be extracted from purely hadronic processes by comparing three-jet production to two-jet production, at various center-of-mass energies. The presence of infrared logarithms in both of these quantities means that this cannot be done sensibly unless both quantities are known to next-to-leading order.

We present here the one-loop matrix elements with five external gluons, which are the hardest part of a $2 \rightarrow 3$ calculation if a traditional diagrammatic method is used. We have performed the calculation using the string-based methods developed in ref. [2] as more efficient tools for one-loop calculations with external gluons. The rules presented there were derived by taking the infinitetension limit of an appropriately-constructed heterotic string amplitude. The structure of the rules can also be understood in conventional field theory [3], and the application of such methods to a calculation such as the present one does not require any knowledge of string theory. (It turns out that it is possible to construct a set of rules yielding more compact integral representation of gluon amplitudes at intermediate stages than would emerge from a straightforward application of the rules in ref. [2]. This alternate set of string-based rules will be discussed elsewhere.)

In the string-based method, one first decomposes the $n$-gluon amplitude, depending on the external momenta, helicities, and color indices $k_{i}, \lambda_{i}$, and $a_{i}$, into sums over certain permutations of color factors, times partial amplitudes, in analogy to the helicity [4,5] and color [6] decomposition of tree amplitudes. At one-loop order in an $S U(N)$ theory, one must also sum over the different spins $J$ of the internal particles; this takes the following form when all internal particles transform as color adjoints,

$$
\mathcal{A}_{n}\left(\left\{k, \lambda_{i}, a_{i}\right\}\right)=\sum_{J} n_{J} \sum_{c=1}^{\lfloor n / 2\rfloor+1} \sum_{\sigma \in S_{n} / S_{n ; c}} \operatorname{Gr}_{n ; c}(\sigma) A_{n ; c}^{[J]}(\sigma)
$$

where $\operatorname{Gr}_{n ; 1}(1)=N \operatorname{Tr}\left(T^{a_{1}} \cdots T^{a_{n}}\right), \operatorname{Gr}_{n ; c}(1)=\operatorname{Tr}\left(T^{a_{1}} \cdots T^{a_{c-1}}\right) \operatorname{Tr}\left(T^{a_{c}} \cdots T^{a_{n}}\right), S_{n}$ is the set of all permutations of $n$ objects, and $S_{n ; c}$ is the subset leaving the trace structure $\mathrm{Gr}_{n ; c}$ invariant. The $T^{a}$ are the set of hermitian traceless $N \times N$ matrices, normalized so that $\operatorname{Tr}\left(T^{a} T^{b}\right)=\delta^{a b}$. For internal particles in the fundamental $(N+\bar{N})$ representation, only the single-trace color structure $(c=1)$ is present, and it is smaller by a factor of $N$. We take in each case a spin- $J$ particle with two states: gauge bosons, Weyl fermions, and complex scalars.

The objects one calculates are the partial amplitudes $A_{n ; c}^{[J]}$, which depend only on the external momenta and helicities. For the five-point function, there is only one independent partial amplitude for each configuration of external helicities; $A_{5 ; 2}$ and $A_{5 ; 3}$ are related to the adjoint contributions to $A_{5 ; 1}$ via decoupling equations [7].

The string-based method meshes well with the spinor helicity representation for the polarization vectors $[4,5]$, which provides an efficient method for extracting the essential gauge-invariant information in an on-shell amplitude. This method yields expressions written in terms of spinor 
products $\langle i j\rangle$ and $[i j]$, which are square roots of Lorentz products $s_{i j}=\left(k_{i}+k_{j}\right)^{2}$ (up to a phase). Unfortunately, the relations - momentum conservation and the Schouten identity - between different forms of a given expression are nonlinear, which makes it hard to give a canonical form for such expressions, or equivalently makes it hard to simplify complicated expressions. However, one can evaluate "phase-invariant" combinations of spinor products in terms of $s_{i j}$ and contractions of the Levi-Civita tensor $\varepsilon(i, j, m, n)=4 i \varepsilon_{\mu \nu \rho \sigma} k_{i}^{\mu} k_{j}^{\nu} k_{m}^{\rho} k_{n}^{\sigma}=[i j]\langle j m\rangle[m n]\langle n i\rangle-\langle i j\rangle[j m]\langle m n\rangle[n i]$. It suffices to calculate the ratios

$$
\frac{\langle i j\rangle[j k]}{\langle i l\rangle[l k]}=\frac{s_{i l} s_{j k}+s_{k l} s_{i j}-s_{i k} s_{j l}-\varepsilon(i, j, k, l)}{2 s_{i l} s_{k l}},
$$

using e.g. methods in reference [8]. In this way spinor products can be eliminated from any expression, apart from an overall prefactor.

For massless five-point kinematics, such an expression can then be written as a rational function in the five kinematic variables $\left\{\beta_{1}, \beta_{2}^{*}, \beta_{3}, \beta_{4}^{*}, \beta_{5}\right\}$ (or any cyclic permutation of this set), where

$$
\beta_{i}=[i, i+1]\langle i+1, i+2\rangle[i+2, i+3]\langle i+3, i\rangle \times\left(-\prod_{j=1}^{5} s_{j, j+1}\right)^{-1 / 2} .
$$

The only independent Levi-Civita contraction is given by $\varepsilon(1,2,3,4) /\left(-\prod_{j=1}^{5} s_{j, j+1}\right)^{1 / 2}=\left(\beta_{5} \beta_{2}^{*}+\right.$ $\left.\beta_{1} \beta_{3}+\beta_{2}^{*} \beta_{4}^{*}+\beta_{3} \beta_{5}+\beta_{4}^{*} \beta_{1}\right) / \beta_{3}=\beta_{i}-\beta_{i}^{*}$ for any $i$, and the independent Lorentz products by $s_{i, i+1}=-1 /\left(\left(\beta_{i}+\beta_{i+1}^{*}\right)\left(\beta_{i+2}+\beta_{i+3}^{*}\right)\right)$. Simplification of rational functions in $\beta_{i}$ is straightforward.

The $\beta_{i}$ variables are related to the variables $\gamma_{i}$ and $\hat{\Delta}_{5}$ used in ref. [9] to perform pentagon integrals, via $\beta_{i}^{(*)}=-\left(\gamma_{i+2} \pm \sqrt{\hat{\Delta}_{5}}\right) / 2$. Indeed, the derivative approach to evaluating tensor integrals [9], when applied to the pentagon integrands encountered in the five-gluon calculation, and expressed in terms of the appropriate set of $\beta_{i}$ variables, allows one to significantly reduce the degree and size of the Feynman parameter polynomials in the integrand.

At tree-level, certain helicity amplitudes vanish identically [10]. The corresponding one-loop amplitudes are then free of infrared divergences. The remaining amplitudes are infrared-divergent; for practical purposes these divergences must be regulated using dimensional regularization. The computation of these helicity amplitudes thus requires the knowledge of five-point loop integrals in $D=4-2 \epsilon[9,11]$.

For the finite helicity amplitudes, supersymmetric identities [12] imply that the contributions of particles of different spin circulating around the loop are related, $A_{n ; c}^{[1]}=-A_{n ; c}^{[1 / 2]}=A_{n ; c}^{[0]}$. (This holds true for the partial amplitudes whether or not the theory as a whole is supersymmetric.) Indeed, in the string-based method, these identities hold for the integrands of each diagram. The amplitudes are

$$
\begin{aligned}
A_{5 ; 1}^{[1]}\left(1^{+}, 2^{+}, 3^{+}, 4^{+}, 5^{+}\right)= & \frac{i}{96 \pi^{2}} \frac{s_{12} s_{23}+s_{23} s_{34}+s_{34} s_{45}+s_{45} s_{51}+s_{51} s_{12}+\varepsilon(1,2,3,4)}{\langle 12\rangle\langle 23\rangle\langle 34\rangle\langle 45\rangle\langle 51\rangle}, \\
A_{5 ; 1}^{[1]}\left(1^{-}, 2^{+}, 3^{+}, 4^{+}, 5^{+}\right)= & \frac{i}{48 \pi^{2}} \frac{1}{[12]\langle 23\rangle\langle 34\rangle\langle 45\rangle[51]}\left[\left(s_{23}+s_{34}+s_{45}\right)[25]^{2}-[24]\langle 43\rangle[35][25]\right. \\
& \left.-\frac{[12][15]}{\langle 12\rangle\langle 15\rangle}\left(\langle 12\rangle^{2}\langle 13\rangle^{2} \frac{[23]}{\langle 23\rangle}+\langle 13\rangle^{2}\langle 14\rangle^{2} \frac{[34]}{\langle 34\rangle}+\langle 14\rangle^{2}\langle 15\rangle^{2} \frac{[45]}{\langle 45\rangle}\right)\right] .
\end{aligned}
$$

In order to present the results for the remaining, infrared-divergent amplitudes in a compact 
form, it is helpful to define the following functions,

$$
\begin{gathered}
\mathrm{L}_{0}(r)=\frac{\ln (r)}{1-r}, \quad \mathrm{~L}_{1}(r)=\frac{\ln (r)+1-r}{(1-r)^{2}}, \quad \mathrm{~L}_{2}(r)=\frac{\ln (r)-(r-1 / r) / 2}{(1-r)^{3}}, \\
\mathrm{Ls}_{1}\left(r_{1}, r_{2}\right)=\frac{1}{\left(1-r_{1}-r_{2}\right)^{2}}\left[\operatorname{Li}_{2}\left(1-r_{1}\right)+\mathrm{Li}_{2}\left(1-r_{2}\right)+\ln r_{1} \ln r_{2}-\frac{\pi^{2}}{6}\right. \\
\left.+\left(1-r_{1}-r_{2}\right)\left(\mathrm{L}_{0}\left(r_{1}\right)+\mathrm{L}_{0}\left(r_{2}\right)\right)\right]
\end{gathered}
$$

where $\mathrm{Li}_{2}$ is the dilogarithm; a prefactor,

$$
c_{\Gamma}=\frac{(4 \pi)^{\epsilon}}{16 \pi^{2}} \frac{\Gamma(1+\epsilon) \Gamma^{2}(1-\epsilon)}{\Gamma(1-2 \epsilon)}
$$

a universal function,

$$
V^{g}=-\frac{1}{\epsilon^{2}} \sum_{j=1}^{5}\left(\frac{\mu^{2}}{-s_{j, j+1}}\right)^{\epsilon}+\sum_{j=1}^{5} \ln \left(\frac{-s_{j, j+1}}{-s_{j+1, j+2}}\right) \ln \left(\frac{-s_{j+2, j-2}}{-s_{j-2, j-1}}\right)+\frac{5}{6} \pi^{2}-\frac{\delta_{R}}{3}
$$

the following functions for the $\left(1^{-}, 2^{-}, 3^{+}, 4^{+}, 5^{+}\right)$helicity configuration,

$$
\begin{aligned}
V^{f} & =-\frac{5}{2 \epsilon}-\frac{1}{2}\left[\ln \left(\frac{\mu^{2}}{-s_{23}}\right)+\ln \left(\frac{\mu^{2}}{-s_{51}}\right)\right]-2, \quad V^{s}=-\frac{1}{3} V^{f}+\frac{2}{9} \\
F^{f}= & -\frac{1}{2} \frac{\langle 12\rangle^{2}(\langle 23\rangle[34]\langle 41\rangle+\langle 24\rangle[45]\langle 51\rangle)}{\langle 23\rangle\langle 34\rangle\langle 45\rangle\langle 51\rangle} \frac{\mathrm{L}_{0}\left(\frac{-s_{23}}{-s_{51}}\right)}{s_{51}} \\
F^{s}= & -\frac{1}{3} \frac{[34]\langle 41\rangle\langle 24\rangle[45](\langle 23\rangle[34]\langle 41\rangle+\langle 24\rangle[45]\langle 51\rangle)}{\langle 34\rangle\langle 45\rangle} \frac{\mathrm{L}_{2}\left(\frac{-s_{23}}{-s_{51}}\right)}{s_{51}^{3}}-\frac{1}{3} F^{f} \\
& -\frac{1}{3} \frac{\langle 35\rangle[35]^{3}}{[12][23]\langle 34\rangle\langle 45\rangle[51]}+\frac{1}{3} \frac{\langle 12\rangle[35]^{2}}{[23]\langle 34\rangle\langle 45\rangle[51]}+\frac{1}{6} \frac{\langle 12\rangle[34]\langle 41\rangle\langle 24\rangle[45]}{s_{23}\langle 34\rangle\langle 45\rangle s_{51}},
\end{aligned}
$$


and the corresponding ones for the $\left(1^{-}, 2^{+}, 3^{-}, 4^{+}, 5^{+}\right)$helicity configuration,

$$
\begin{aligned}
& V^{f}=-\frac{5}{2 \epsilon}-\frac{1}{2}\left[\ln \left(\frac{\mu^{2}}{-s_{34}}\right)+\ln \left(\frac{\mu^{2}}{-s_{51}}\right)\right]-2, \quad V^{s}=-\frac{1}{3} V^{f}+\frac{2}{9} \\
& F^{f}=-\frac{\langle 13\rangle^{2}\langle 41\rangle[24]^{2}}{\langle 45\rangle\langle 51\rangle} \frac{\operatorname{Ls}_{1}\left(\frac{-s_{23}}{-s_{51}}, \frac{-s_{34}}{-s_{51}}\right)}{s_{51}^{2}}+\frac{\langle 13\rangle^{2}\langle 53\rangle[25]^{2}}{\langle 34\rangle\langle 45\rangle} \frac{\operatorname{Ls}_{1}\left(\frac{-s_{12}}{-s_{34}}, \frac{-s_{51}}{-s_{34}}\right)}{s_{34}^{2}} \\
& -\frac{1}{2} \frac{\langle 13\rangle^{3}(\langle 15\rangle[52]\langle 23\rangle-\langle 34\rangle[42]\langle 21\rangle)}{\langle 12\rangle\langle 23\rangle\langle 34\rangle\langle 45\rangle\langle 51\rangle} \frac{\mathrm{L}_{0}\left(\frac{-s_{34}}{-s_{51}}\right)}{s_{51}} \\
& F^{s}=-\frac{\langle 12\rangle\langle 23\rangle\langle 34\rangle\langle 41\rangle^{2}[24]^{2}}{\langle 45\rangle\langle 51\rangle\langle 24\rangle^{2}} \frac{2 \mathrm{Ls}_{1}\left(\frac{-s_{23}}{-s_{51}}, \frac{-s_{34}}{-s_{51}}\right)+\mathrm{L}_{1}\left(\frac{-s_{23}}{-s_{51}}\right)+\mathrm{L}_{1}\left(\frac{-s_{34}}{-s_{51}}\right)}{s_{51}^{2}} \\
& +\frac{\langle 32\rangle\langle 21\rangle\langle 15\rangle\langle 53\rangle^{2}[25]^{2}}{\langle 54\rangle\langle 43\rangle\langle 25\rangle^{2}} \frac{2 \mathrm{Ls}_{1}\left(\frac{-s_{12}}{-s_{34}}, \frac{-s_{51}}{-s_{34}}\right)+\mathrm{L}_{1}\left(\frac{-s_{12}}{-s_{34}}\right)+\mathrm{L}_{1}\left(\frac{-s_{51}}{-s_{34}}\right)}{s_{34}^{2}} \\
& +\frac{2}{3} \frac{\langle 23\rangle^{2}\langle 41\rangle^{3}[24]^{3}}{\langle 45\rangle\langle 51\rangle\langle 24\rangle} \frac{\mathrm{L}_{2}\left(\frac{-s_{23}}{-s_{51}}\right)}{s_{51}^{3}}-\frac{2}{3} \frac{\langle 21\rangle^{2}\langle 53\rangle^{3}[25]^{3}}{\langle 54\rangle\langle 43\rangle\langle 25\rangle} \frac{\mathrm{L}_{2}\left(\frac{-s_{12}}{-s_{34}}\right)}{s_{34}^{3}} \\
& +\frac{\mathrm{L}_{2}\left(\frac{-s_{34}}{-s_{51}}\right)}{s_{51}^{3}}\left(\frac{1}{3} \frac{\langle 13\rangle[24][25](\langle 15\rangle[52]\langle 23\rangle-\langle 34\rangle[42]\langle 21\rangle)}{\langle 45\rangle}\right. \\
& \left.+\frac{2}{3} \frac{\langle 12\rangle^{2}\langle 34\rangle^{2}\langle 41\rangle[24]^{3}}{\langle 45\rangle\langle 51\rangle\langle 24\rangle}-\frac{2}{3} \frac{\langle 32\rangle^{2}\langle 15\rangle^{2}\langle 53\rangle[25]^{3}}{\langle 54\rangle\langle 43\rangle\langle 25\rangle}\right) \\
& +\frac{1}{6} \frac{\langle 13\rangle^{3}(\langle 15\rangle[52]\langle 23\rangle-\langle 34\rangle[42]\langle 21\rangle)}{\langle 12\rangle\langle 23\rangle\langle 34\rangle\langle 45\rangle\langle 51\rangle} \frac{\mathrm{L}_{0}\left(\frac{-s_{34}}{-s_{51}}\right)}{s_{51}}+\frac{1}{3} \frac{[24]^{2}[25]^{2}}{[12][23][34]\langle 45\rangle[51]} \\
& -\frac{1}{3} \frac{\langle 12\rangle\langle 41\rangle^{2}[24]^{3}}{\langle 45\rangle\langle 51\rangle\langle 24\rangle[23][34] s_{51}}+\frac{1}{3} \frac{\langle 32\rangle\langle 53\rangle^{2}[25]^{3}}{\langle 54\rangle\langle 43\rangle\langle 25\rangle[21][15] s_{34}}+\frac{1}{6} \frac{\langle 13\rangle^{2}[24][25]}{s_{34}\langle 45\rangle s_{51}} \text {. }
\end{aligned}
$$

For positive values of $s_{i j}$, the logarithms and dilogarithms develop imaginary parts according to the prescription $s_{i j} \rightarrow s_{i j}+i \varepsilon$. We also remind the reader of the tree amplitudes, $A_{5}^{\text {tree }}\left(1^{-}, 2^{-}, 3^{+}, 4^{+}, 5^{+}\right)=$ $i\langle 12\rangle^{4} /(\langle 12\rangle\langle 23\rangle\langle 34\rangle\langle 45\rangle\langle 51\rangle)$ and $A_{5}^{\text {tree }}\left(1^{-}, 2^{+}, 3^{-}, 4^{+}, 5^{+}\right)=i\langle 13\rangle^{4} /(\langle 12\rangle\langle 23\rangle\langle 34\rangle\langle 45\rangle\langle 51\rangle)$.

In terms of these functions, the $\overline{\mathrm{MS}}$ renormalized amplitudes are

$$
\begin{aligned}
A_{5 ; 1}^{[0]} & =c_{\Gamma}\left(V^{s} A_{5}^{\text {tree }}+i F^{s}\right), \\
A_{5 ; 1}^{[1 / 2]} & =-c_{\Gamma}\left(\left(V^{f}+V^{s}\right) A_{5}^{\text {tree }}+i\left(F^{f}+F^{s}\right)\right), \\
A_{5 ; 1}^{[1]} & =c_{\Gamma}\left(\left(V^{g}+4 V^{f}+V^{s}\right) A_{5}^{\text {tree }}+i\left(4 F^{f}+F^{s}\right)\right) .
\end{aligned}
$$

The rest of the helicity amplitudes are related by cyclic permutations or complex conjugation to those given above. It is interesting to note that in supersymmetric theories, the $V^{s}$ and $F^{s}$ terms cancel out of the final amplitude, and that in $N=4$ supersymmetric theories only the $V^{g}$ term survives. The separation implied above into $g, f$, and $s$ pieces arises naturally on a diagram-bydiagram basis within the string-based approach. In this approach the $V^{g}$ term represents the only calculational difference between the contributions with gluons circulating around the loop, and those with fermions; this term has a particularly simple expression at every intermediate stage of the calculation. The parameter $\delta_{R}$ controls the variant of dimensional regularization scheme [2]: 
for $\delta_{R}=0$, one obtains the four-dimensional helicity scheme, while for $\delta_{R}=1$ one obtains the 't Hooft-Veltman scheme.

There are several checks we have applied. We have checked gauge invariance, both by computing amplitudes with longitudinal gluons, verifying that one obtains zero, and by calculating a helicity amplitude with an alternate choice of spinor-helicity reference momenta, and verifying that the result is unchanged. In addition, the forms given above display manifestly the reflection symmetries expected of the amplitudes, symmetries that are not present in the contributions of the individual diagrams. The amplitudes also have consistent limits as one of the gluon momenta becomes soft, and as two adjacent momenta become collinear.

At next-to-leading order, only the infrared-divergent helicity amplitudes (5-10) enter into the construction of a program for physical quantities. In order to construct such a program for three-jet quantities, one must form the interference of the tree amplitude with the loop amplitude; this has the form $[7]$

$$
\begin{aligned}
\sum_{\text {colors }}\left[\mathcal{A}_{5}^{*} \mathcal{A}_{5}\right]_{\mathrm{NLO}} & =2 g^{8} N^{4}\left(N^{2}-1\right)\left[\operatorname{Re} \sum_{\sigma \in S_{5} / Z_{5}} A_{5}^{\text {tree } *}(\sigma) A_{5 ; 1}(\sigma)\right. \\
& +\frac{1}{N^{2}} \operatorname{Re} \sum_{\rho \in S_{5} / Z_{5}}\left[A_{5}^{\text {tree } *}(r \cdot \rho) A_{5 ; 1}(\rho)-A_{5}^{\text {tree } *}(\rho) A_{5 ; 1}(r \cdot \rho)\right] \\
& \left.+\frac{2}{N^{2}} \operatorname{Re} \sum_{h \in H_{5}} \sum_{p \in P\left(\begin{array}{l}
5 \\
3
\end{array}\right)} A_{5}^{\text {tree } *}(h \cdot p) A_{5 ; 3}(p)\right],
\end{aligned}
$$

where $r$ is the permutation $(24135) ; P\left(\begin{array}{l}5 \\ 3\end{array}\right)$ is the ten-element set of distinct partitions of five elements into lists of length two and three; and $H_{5}=\{(12345),(34125),(31245),(21345),(32145)$, $(34215)\}$. For QCD with $n_{f}$ flavors of massless quarks, one substitutes $A_{5 ; 1} \rightarrow A_{5 ; 1}^{[1]}+\frac{n_{f}}{N} A_{5 ; 1}^{[1 / 2]}$ and $A_{5 ; 3} \rightarrow A_{5 ; 3}^{[1]}$ into equation (11). One must then combine this virtual correction with the singular terms in the $2 \rightarrow 4$ matrix elements arising from the integration over soft and collinear phase space. The Giele-Glover formalism [13] makes use of the color ordering in construction of universal functions representing the results of the soft and collinear integrations, and is the most convenient one for doing so. We have used it to check that the poles in $\epsilon$ do cancel as expected. 


\section{References}

[1] R. K. Ellis and J. C. Sexton, Nucl. Phys. B269:445 (1986).

[2] Z. Bern and D. A. Kosower, Phys. Rev. Lett. 66:1669 (1991); Nucl. Phys. B379:451 (1992).

[3] Z. Bern and D. C. Dunbar, Nucl. Phys. B379:562 (1992).

[4] F. A. Berends, R. Kleiss, P. De Causmaecker, R. Gastmans, and T. T. Wu, Phys. Lett. 103B:124 (1981);

P. De Causmaeker, R. Gastmans, W. Troost, and T. T. Wu, Nucl. Phys. B206:53 (1982);

R. Kleiss and W. J. Stirling, Nucl. Phys. B262:235 (1985);

J. F. Gunion and Z. Kunszt, Phys. Lett. 161B:333 (1985);

R. Gastmans and T.T. Wu, The Ubiquitous Photon: Helicity Method for QED and QCD (Clarendon Press) (1990);

M. Mangano and S. J. Parke, Phys. Rept. 200:301 (1991).

[5] Z. Xu, D.-H. Zhang, L. Chang, Tsinghua University preprint TUTP-84/3 (1984), unpublished;

Z. Xu, D. Zhang, L. Chang, Nucl. Phys. B291:392 (1987).

[6] F. A. Berends and W. T. Giele, Nucl. Phys. B294:700 (1987);

M. Mangano, S. J. Parke and Z. Xu, Nucl. Phys. B298:653 (1988); Nucl. Phys. B299:673 (1988);

D. Zeppenfeld, Int. J. Mod. Phys. A3:2175 (1988).

[7] Z. Bern and D. A. Kosower, Nucl. Phys. B362:389 (1991).

[8] H. W. Fearing and R. R. Silbar, Phys. Rev. D6:471 (1971).

[9] Z. Bern, L. Dixon, and D. A. Kosower, preprints SLAC-PUB-6001 and SLAC-PUB-5947.

[10] S. J. Parke and T. R. Taylor, Phys. Rev. Lett. 56:2459 (1986).

[11] R. K. Ellis, W. T. Giele and E. Yehudai, in preparation.

[12] M. T. Grisaru and H. N. Pendleton, Nucl. Phys. B124:81 (1977).

[13] W. T. Giele and E. W. N. Glover, Phys. Rev. D46:1980 (1992);

W. T. Giele, E. W. N. Glover, and D. A. Kosower, preprint Fermilab-Pub-92/230-T. 